\title{
Optimization in Smart Grids and Homes Smart and Easy Way to Select the Best!!
}

\author{
Tejas Gujrati
}

\begin{abstract}
In this paper, an optimized concepts to be applied in homes and other commercial places has been discussed. These concepts will not only help the consumers to have a fixed utilization of power (reference chosen) but it will ensure the grid about the maximum utilization (below maximum limit) that can be used by this type of particular homes. Various concepts of optimization to minimize the cost of purchase of power has been discussed. The human interactive device has been discussed which will provide a feedback to the people about the electricity used till time in a day and enable him/her to use a fixed amount of power and at a specific time to maximize the time of utilization at a minimum cost. This device will not only provide information but perform certain task automatically to ensure best that could be done at that particular time.
\end{abstract}

Index Terms-Sensors; Wireless device website; Wireless communication; Meters; Energy consumptions; Optimized output.

\section{INTRODUCTION}

Optimization refers to the process of selecting the best solution among the possible alternatives. And we are working on same process to get the best possible output according to the reference chosen. This method will not only help the consumers in managing their electricity bills but also helps the grids to manage loads at the particular instance of time in case of need. This will also help to manage the production in power depending upon the communication between the homes and the grids or production with the help of this device. The main problem of running the generators in generating stations at no load and the losses related to it can be eliminated. Also the data related to the power produced by the private sectors will also be updated and will be well known to the generations so that they will be well aware of the fact that the power supplied to the grids are sufficient enough to manage the present load in that area. In brief we can say that through this device every section from power production to power consumption including the transmission will be well aware of the fact what is happening in the electricity world.

In the time of increase in load suddenly the device will alarm the remaining percentage of electricity that can be still be used with the particular load and will provide the utilization time of this load ,before this time if the load is removed the device will recalculate the remaining time on grid or it will cut off the supply from grid and switch automatically to another available smart source smartly, that is in day time either solar, wind or

Tejas Gujrati is with the Electrical Department, Yildiz Technical University Davutpaşa, Istanbul, Turkey . battery but at night mostly preferred sources are battery or wind. If the user still have to use the load from the grid the information is sent to the grid and it is updated globally to all the sections and thus the generation will check whether this load can be handled by the private sector productions or other third party production, if yes then it is supplied or the generation can be increased this method can be implemented to a particular area and correspondingly to a city. If the user don't have to use the grid power he can still opt for generator other sources. In case the user have used up all his selected electricity before the date (he will be alarmed each day and at specific time) then we will have the reset option to his selected reference amount and he can able to input a new reference amount which will be updated globally via website.

\section{DESCRIPTION OF WORK}

As we know, the equation for minimizing the cost is given by:

$$
T C=\sum_{t}\left(P_{t}^{g r i d} \cdot \Delta T \cdot \lambda_{t}^{b u y}\right)
$$

Where:

TC is minimized cost.

$\mathrm{P}_{\mathrm{t}}{ }^{\text {grid }}=$ power exchange with the grid in time $\mathrm{t}$.

deltaT $=$ change in time

$\lambda$

buy= price of energy exchange with the grid in time t.

In this context, we are trying to make the cost element (TC) constant. And we are taking the input to the device $\mathrm{P}_{t}^{\mathrm{g} \text { rid }}$ accordingly which will control the operation of all the devices in the home on the basis of reference chosen. At the same time this device will send the output to the website globally and will keep the distribution and generation system updated. This device will be attached just after the meter in the home with the connection of all the device in the home along with an indicating LED across each device which will tell the user about the best time to use the heavy electronics like washing machine, air conditioner, etc. This device will be updating periodically and letting the user to use the maximum electricity at the best time according to cost. Not only this, it will provide information to the user to switch to the natural source to save some bucks by collecting the data from the current load utilization and the cost of per unit of electricity. The website will keep the generation as well as distribution systems updated about the present and future need of the consumer and can accordingly plan for productions. In case of any emergency 
demand the user can update his need on the website and this need will be available to the productions or in case the user have used us all his electricity unit according to the provided cost he will be given the option to reset his reference and select a new pack for remaining days. This process will not only help the consumers but it will save the time of the government people who spend their valuable time checking the meter and generating the bills accordingly.

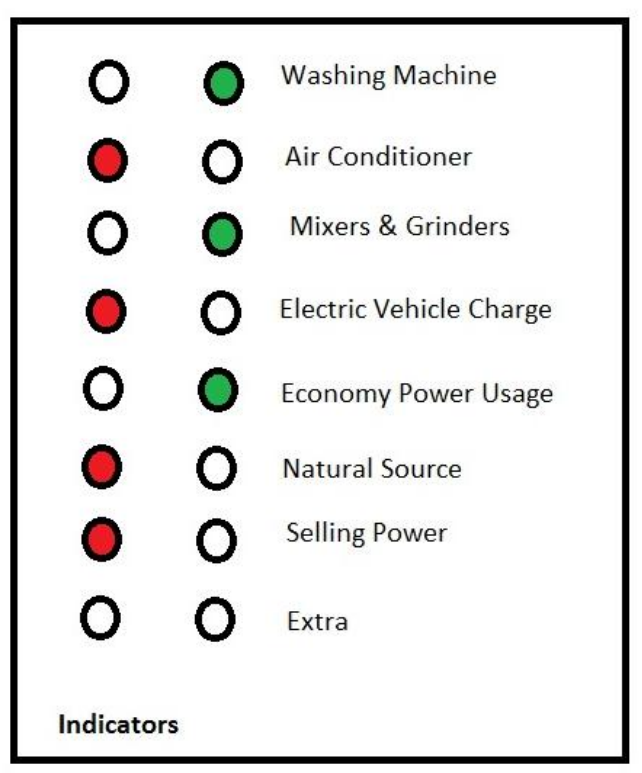

Fig.1: Indicators representing favourable switching options

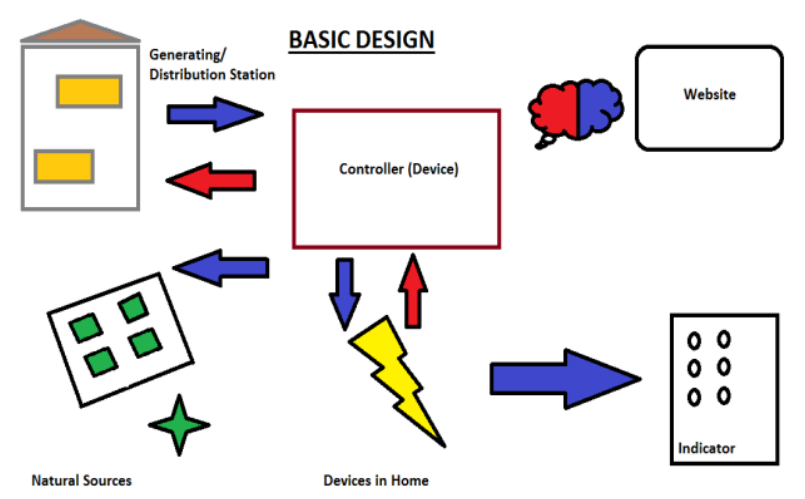

Fig. 2: Basic Design of Operation

The above figure explains the basic design of the Smart home, the controller will be connected such that it can communicate with generating station and it the same time collect the information from the station and display it on the website, also this device will collect the data from the website and take necessary actions like switching which not only involves the heavy electronics of the homes but it will be capable to switch to natural sources of energy at the time of need (specially at the times of peak to save bucks), the device like fans, lights will not make huge difference unless a remarkable number of them is used so if the customer is so much concerned about his bill he can take indicators for that too otherwise these values will be included with the values of heavy electronics.

The website is designed to provide all the necessary information to the user as well as to the device. The customer is required to login into his/her account and once he do that he can now access the details like his tariff chosen for one month, units allotted to him for a month according to his tariff (less than the maximum limit), the units consumed my him, current load of his home and also the days left according to his tariff on the current load. The website will also have the links for changing the reference amount of his tariff, in case the customer has used up all his units allotted and also a pre form for emergency demand in case of special occasion or demand in home. The device is intelligent enough to choose among the various natural sources of energy available and will switch automatically if programmed accordingly. Also, the electricity rates will be applied according to the rates of government which will be taken from the website according the units used and updated.

\section{CALCULATIONS}

Assuming the situation in India, and the customer has used up his first 200 units of electricity so now according to the government rates he will be charged at Rs.4.80 per unit. So,

Tariff for 1 month chosen $=$ Rs 5000

1 unit $=$ Rs 3.70

Units used $=200$

Bucks remaining $=$ Rs 4260

Now if the current load in home is 1000 watts $=1 \mathrm{Kw}$.

Cost of electricity $=$ Rs 4.80

According to tariff, (if 887.5 unit is used for $1 \mathrm{hr}$ the tariff will be exhausted)

So, if the same 1000 watts is used the electricity will last up to approximately 887.5 hours or 37 days.

Note- The above calculations are made for non- peak hours.

\section{A. Details about the device}

It is the mastermind that analysis and take almost all the decisions by itself. It is an IOT device that is capable of collecting the data from the server and the same time sending the data to the server that is this device will have an access to server. The server data will be processed in the controller and according to the reference set the actions will be performed. Along with it can control all the electrical and electronic devices of the home and if programmed to switch, it can switch from non-renewable to renewable source of energy in peak hours, also these device will alarm the customer of the updation in the consumption and also the change of cost. This device will be connected just after the meter device and the output of this device will be shown in the indicator. 


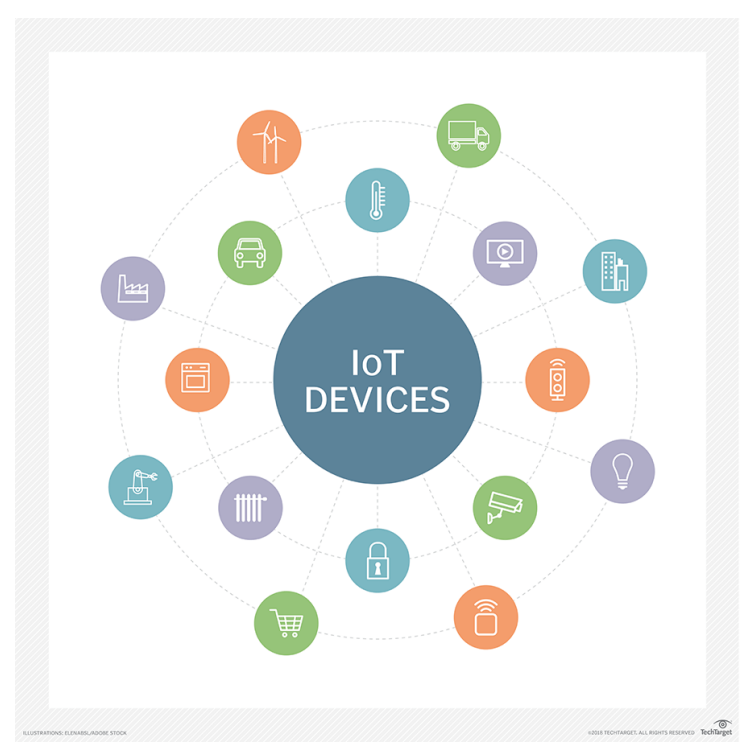

Fig. 3: IOT devices (Internet of Things)

"IoT devices, or any of the many things in the internet of things, are nonstandard computing devices that connect wirelessly to a network and have the ability to transmit data. IoT involves extending internet connectivity beyond standard devices, such as desktops, laptops, smartphones and tablets, to any range of traditionally dumb or non-internet-enabled physical devices and everyday objects. Embedded with technology, these devices can communicate and interact over the internet, and they can be remotely monitored and controlled.

\section{B. IoT device examples and applications}

Connected devices are part of a scenario in which every device talks to other related devices in an environment to automate home and industry tasks, and to communicate usable sensor data to users, businesses and other interested parties. IoT devices are meant to work in concert for people at home, in industry or in the enterprise. As such, the devices can be categorized into three main groups: consumer, enterprise and industrial.

Consumer connected devices include smart TVs, smart speakers, toys, wearables and smart appliances. Smart meters, commercial security systems and smart city technologies -such as those used to monitor traffic and weather conditions -are examples of industrial and enterprise IoT devices. Other technologies, including smart air conditioning, smart thermostats, smart lighting and smart security, span home, enterprise and industrial uses.

In a smart home, for example, a user arrives home and his car communicates with the garage to open the door. Once inside, the thermostat is already adjusted to his preferred temperature, and the lighting is set to a lower intensity and his chosen color for relaxation, as his pacemaker data indicates it has been a stressful day. In the enterprise, smart sensors located in a conference room can help an employee locate and schedule an available room for a meeting, ensuring the proper room type, size and features are available. When meeting attendees enter the room, the temperature will adjust according to the occupancy, and the lights will $\operatorname{dim}$ as the appropriate
PowerPoint loads on the screen and the speaker begins his presentation.

\section{IoT device connectivity and networking}

The networking, communication and connectivity protocols used with internet-enabled devices largely depend on the specific IoT application deployed. Just as there are many different IoT applications, there are many different connectivity and communications options. Communications protocols include CoAP, DTLS and MQTT, among others. Wireless protocols include IPv6, LPWAN, ZigBee, Bluetooth Low Energy, Z-Wave, RFID and NFC. Cellular, satellite, Wi-Fi and Ethernet can also be used. Each option has its tradeoffs in terms of power consumption, range and bandwidth, all of which must be considered when choosing connected devices and protocols for a particular IoT application. To share the sensor data they collect, IoT devices connect to an IoT gateway or another edge device where data can either be analyzed locally or sent to the cloud for analysis."

\section{Impact to Society}

1) The problems of the people related to high bill issue will be resolved.

2) It will help people to have a better energy management.

3) It will save time of the government employees by directly uploading the number of unit consumed by the customer in a month and also the money to be paid.

4) It will eliminate a lot of errors that arises related to bills.

5) This process and device can be expanded from an area to a city and then to the state.

6) The consumption during the peak hours can be reduced.

7) The device can help the customer by keeping them updated about the cost of electricity and non-peak hours.

8) Emergency demand will be well known to the distribution or generation system and so arrangements can be made accordingly.

9) This project will contribute toward the evolution of Smart Homes in an optimized and smart way.

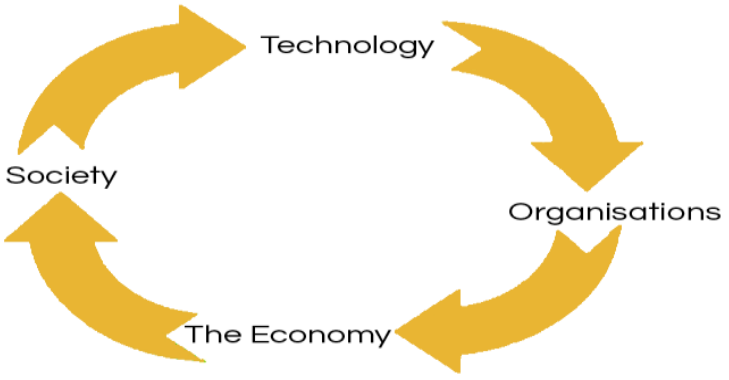

Fig. 4: Impact to society 


\section{CONCLUTION OUTPUT DESCRIPTION AND RESUlt}

\section{Electricity Board}

\section{Optimization Portal}

Area name : Area 51

Customer name : Manhattan

Bill no : F3221515

Tarif for one month : \$xyz

Units allotted : 500 Units

Units consumed : 150 Units

Current Load : 500 Units

Days left on current load : ** Days

Fig. 5: The Central view of website

\section{Emergençy demand(portal).}

Go to Settings to activate Windows.

Fig. 6: Down right portion of website

\section{$\underline{\text { Requests }}$}

\section{Change reference amount}

Fig.7: The leftmost down portion of website

The upper left most portion of the website will be showing dates, days and will be updated periodically. The underlined strings are the links which will open to a form for requests, changing the reference amount will set a new reference value and the used units and used amounts will be added with this new references.

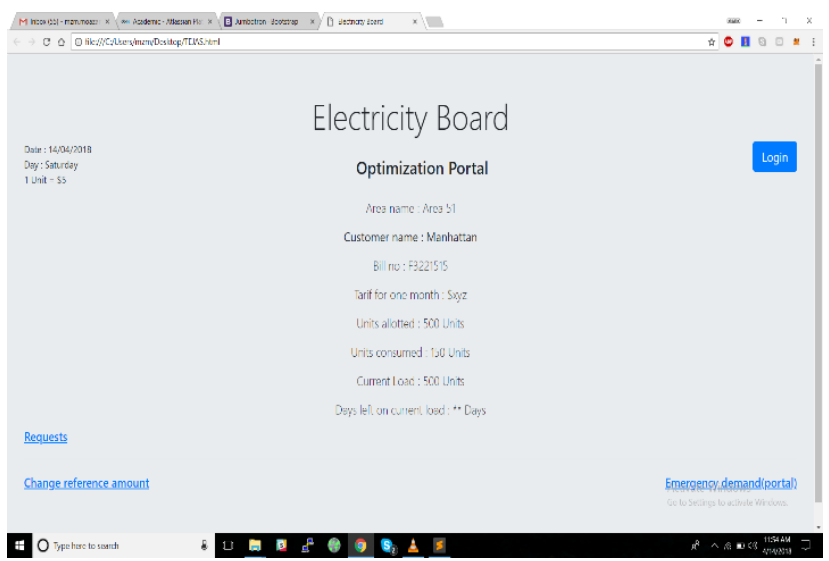

Fig. 8: The website view
The above figures represent the contents of the website and how will it look like. The above figure also define the prototype view of the website. And the website is dynamic and responsive.

\section{ACKNOWLEDGMENT}

I would like to thank my teacher "Professor. Ozan ERDINÇ" for giving such a remarkable platform which tend me to think about something new and innovative in between the intensive course schedule. Also, I would like to thank my friends for motivating and supporting me.

\section{REFERENCES}

[1] F. Akyildiz, X. Wang, and W. Wang, "Wireless mesh networks: a survey," vol. 47, pp. 445-487, 2005.

[2] J. Ekanayake and K. Liyanage, Smart Grid Technology and Applications, First edit. New Delhi, 2012

[3] M. Vega, F. Santamaria, and E. Rivas, "Modeling for home electric energy management: A review," Renew. Sustain. Energy Rev., vol. 52, pp. 948-959, 2015. https://doi.org/10.1016/j.rser.2015.07.023

[4] T. D. P. Mendes, R. Godina, E. M. G. Rodrigues, J. C. O. Matias, and J. P. $\mathrm{S}$. Catalao, "Smart and energy-efficient home implementation: Wireless communication technologies role," Int. Conf. Power Eng. Energy Electr. Drives, vol. 2015-Septe, pp. 377-382, 2015 https://doi.org/10.1109/PowerEng.2015.7266346

[5] J. Byun, B. Jeon, J. Noh, Y. Kim, and S. Park, "An Intelligent Self-Adjusting Sensor for Smart Home Services based on ZigBee Communications," vol. 58, no. 3, pp. 794-802, 2012.

[6] M. B. Yassein, W. Mardini, and A. Khalil, Smart Homes Automation using Z-wave Protocol," 2016. 